\title{
DOPED CONTACTS FOR HIGH-LONGEVITY OPTICALLY ACTIVATED, HIGH GAIN GaAs PHOTOCONDUCTIVE SEMICONDUCTOR SWITCHES
}

\author{
A. Mar, G. M. Loubriel, F. J. Zutavern, M. W. O’Malley, W. D. Helgeson, \\ D. J. Brown, H. P. Hjalmarson, and A. G. Baca \\ Sandia National Laboratories, Albuquerque, New Mexico 87185-1153 \\ R. L. Thornton, R. D. Donaldson, \\ Xerox Palo Alto Research Center, Palo Alto, California 94304
}

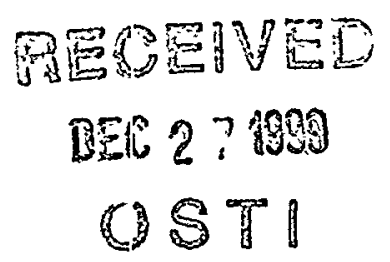

\section{ABSTRACT}

The longevity of high gain GaAs photoconductive semiconductor switches (PCSS) has been extended to over 100 million pulses. This was achieved by improving the ohmic contacts through the incorporation of a doped layer that is very effective in the suppression of filament formation, alleviating current crowding. Damage-free operation is now possible with virtually infinite expected lifetime at much higher current levels than before. The inherent damage-free current capacity of the bulk GaAs itself depends on the thickness of the doped layers and is at least $100 \mathrm{~A}$ for a dopant diffusion depth of $4 \mu \mathrm{m}$. The contact metal has a different damage mechanism and the threshold for damage ( $40 \mathrm{~A})$ is not further improved beyond a dopant diffusion depth of about $2 \mu \mathrm{m}$. In a diffusion-doped contact switch, the switching performance is not degraded when contact metal erosion occurs, unlike a switch with conventional contacts. This paper will compare thermal diffusion and epitaxial growth as approaches to doping the contacts. These techniques will be contrasted in terms of the fabrication issues and device characteristics.

\section{BACKGROUND}

The subject device of this paper is the optically triggered, high-gain lateral GaAs photoconductive switch (PCSS). They are constructed using semi-insulating (SI) GaAs of high resistivity $>10^{7} \Omega-\mathrm{cm}$ and metal contacts that are used to connect the switch to an energy source and a load. At electric fields above 4 to $6 \mathrm{kV} / \mathrm{cm}$ these switches exhibit high gain. ${ }^{1}$ In the "on" state the field across the switch stabilizes to a constant called the lockon field. During high gain switching, the PCSS emit bandgap radiation by carrier recombination. This radiation under high gain operation, when imaged, is in the form of filaments. ${ }^{2}$

Although such high-gain operation is useful for many applications, the filamentary nature of this current impacts negatively the operational lifetime of the switches. This is manifested in the form of damage of the semiconductor to metal interface that initially appears as a trench, metal erosion, or damage in the GaAs away from the contacts (in the gap between the contacts). Contact erosion is an important damage mechanism since it is the main cause of degradation of switching characteristics, resulting in higher on-state resistance and voltage drop, and ultimately the ceasing of switch function.

\section{OPTICALLY-INDUCED DIFFUSE AND MULTIPLE FILAMENT'S}

Damage to the PCSS contacts occurs due to current crowding at the point of filament termination at the edge of the contact. This is exacerbated by the difficulty in making low-resistance ohmic contacts to SI GiaAs. We have previously shown ${ }^{3}$ that the trigger light can be applied to diffuse the filaments near the contacts, improving the current distribution and longevity. Another refinement of this technique we have investigated is multiple filaments using multiple line triggering: as shown in Figure 1.

In this example we have used trigger light irnaged into 8 lines across the width of the switch to initiate: 8 distinct filaments that the current will be distributed amongst. When triggered in this manner, switching at $400 \mathrm{~A}$ results in current drop of only $2 \%$ after 100 shots. The same 


\section{DISCLAIMER}

This report was prepared as an account of work sponsored by an agency of the United States Government. Neither the United States Government nor any agency thereof, nor any of their employees, make any warranty, express or implied, or assumes any legal liability or responsibility for the accuracy, completeness, or usefulness of any information, apparatus, product, or process disclosed, or represents that its use would not infringe privately owned rights. Reference herein to any specific commercial product, process, or service by trade name, trademark, manufacturer, or otherwise does not necessarily constitute or imply its endorsement, recommendation, or favoring by the United States Government or any agency thereof. The views and opinions of authors expressed herein do not necessarily state or reflect those of the United States Government or any agency thereof. 


\section{DISCLAIMER}

Portions of this document may be illegible in electronic image products. Images are produced from the best available original document. 


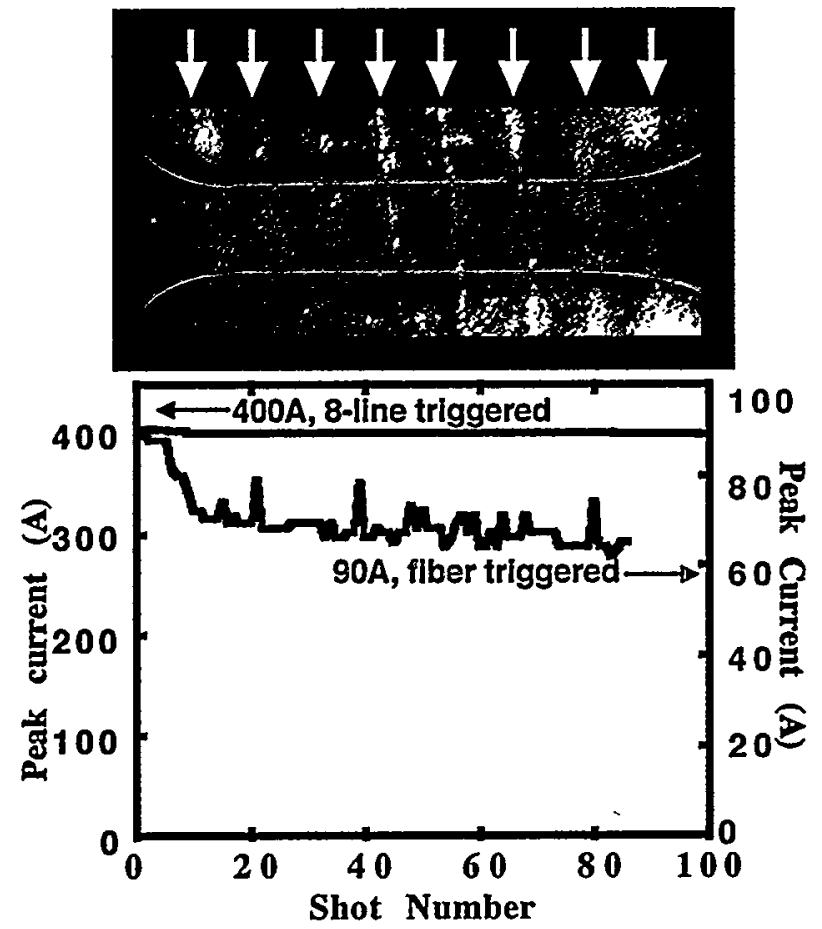

Figure 1. Switch lifetime improvement using multi-line vs. fiber spot triggering.

switch operating at only $90 \mathrm{~A}$ shows a $28 \%$ current drop when fiber (spot) triggered.

\section{DOPED CONTACT FABRICATION}

Due to the improvements in current distribution and lifetime demonstrated by the optical generation of carriers near the contacts, we have sought to incorporate doped layers with high carrier concentrations under the contact regions to reduce the contact resistance and spread the current, as shown in Figure 2. The doped regions also serve to transfer the point of filament termination from the metal - semiconductor interface to a doped semiconductor - semiconductor interface that is more robust against damage. Another embodiment of this idea under investigation involves the use of a thick, less conductive layer grown on top of the highly doped layer. This serves to strongly favor lateral current spreading as this vertical resistance serves to equalize the voltage drops among different current paths from the switch gap to different points on the metal contact. Such a "current leveling layer" has been demonstrated in vertical cavity semiconductor lasers to improve current injection uniformity in edge-injected structures. ${ }^{4}$ Two main approaches for the fabrication of switches with doped
Conventional switch:

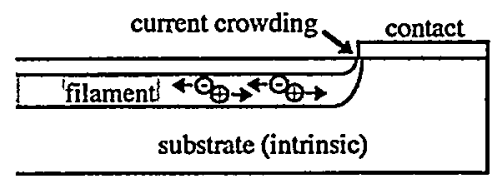

Switch with doped contact layer:

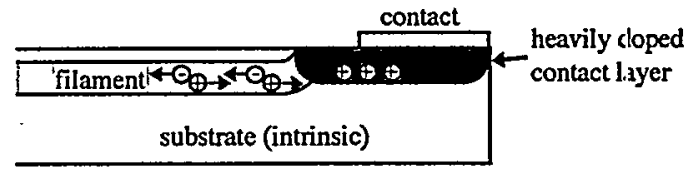

Switch w/doped contact and resistive current-leveling layers:

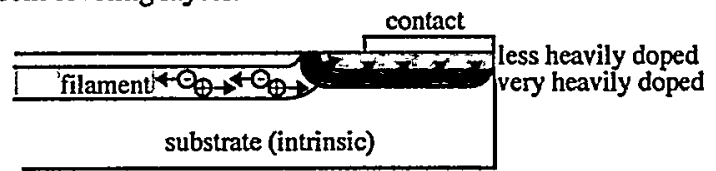

Figure 2. Current crowding and effect of doped contacts.

contact layers are being developed. The first is epitaxial growth using MOCVD to incorporate grown layers of highly doped material under the contacts. In one such approach, n-type (Si-doped) material is first deposited over the entire wafer and then etched away except in the regions where the switch cathodes are formed. This process is then repeated for a p-type growth (7zn-doped) for the anodes and then ohmic contacts are made to the doped material. Because this process results in a nonplanar surface, another preferred approach under development utilizes patterned epitaxial regrowh. In this process, the contact regions are etched to somi? depth (a few microns) and highly doped GaAs is then regrown into these regions by the same thickness as the etch depth using a growth mask $\left(\mathrm{SiO}_{\mathrm{x}}\right)$ that prevents growth elsewhere. This process is also repeated for the two different contact polarities and ohmic contact: made to the doped layers, which results in a planar structure. Because of non-idealities associated with the patterned regrowth, this process is still in development to overcome the issues of conductive material grown on the growth mask (into the switch gaps), and growth non-plariarity.

The second technique we are pursuing for doped PCSS contacts is dopant diffusion. In this prozess, $\mathrm{SiN}_{\mathbf{x}}$ is deposited and patterned to serve as a diffusion mask. An Si source layer and $\operatorname{SiN}_{x}$ encapsulation layer are then deposited and the Si driven in using a tube furnace at $800^{\circ} \mathrm{C}$. These layers are left in place and patterned to serve as a diffusion mask for $\mathrm{Zn}$. Vaporixed $\mathrm{Zn}$ is 
diffused into the substrate in an open tube furnace at approximately $600^{\circ} \mathrm{C}$. Further details regarding the diffusion processing can be found in reference [5]. Ohmic contacts are then made to the doped regions to complete the fabrication. A microphotograph of such a completed device is shown in Figure 3.

\section{PERFORMANCE OF PCSS WITH DOPED CONTÁCTS}

The recombination radiation in PCSS filaments affords the ability to image them using an IR sensitive video camera. Such imagery of a filament in a PCSS with doped contacts is shown in Figure 4. The image shows that the doped layers are highly effective in the suppression of the filament formation near the contacts. This filament suppression at the contact is effective for peak current levels of up to. approximately $40-60 \mathrm{~A}$. In this regime, damage-free operation of the switch is obtained and lifetime is expected to be virtually infinite. In such a case at $80 \mathrm{~A}, 2$ million shots were fired with no detectable damage. At 23A, we obtained 100 million shots at which point the test was stopped, although the switch was still operating. Because high shot count longevity tests for such operation have become extremely tedious, our tests now typically are conducted to 100,000 shots. The switch is then characterized for the presence of any detectable damage under high magnification.

At sufficiently high currents, the filament is not sufficiently suppressed in the doped region to prevent damage to the contact. The nature of this damage and its effects however, is markedly different from that in a conventional PCSS. In the past, longevity tests have determined that damage due to current crowding in conventional PCSS contacts occurs first as a void or trench in the GaAs at the edge of the undoped contacts. Presumably due to the lower mobility of holes in GaAs, more severe damage occurs first at the p-contact of the PCSS. At higher currents and shot counts, this damage is accompanied by damage to the contact metal, in the form of erosion or loss of adhesion to the GaAs. These types of damage are shown in Figure 5. In the case of dopedlayer contacts, this trend is reversed, where damage to the metal occurs at lower currents than it takes to damage the bulk material. In a conventional switch, this causes increased voltage drop and decreased current as the metal erosion occurs. This is not the case with PCSS with doped contacts, where the point of filament termination is on the doped region and the switching behavior remains

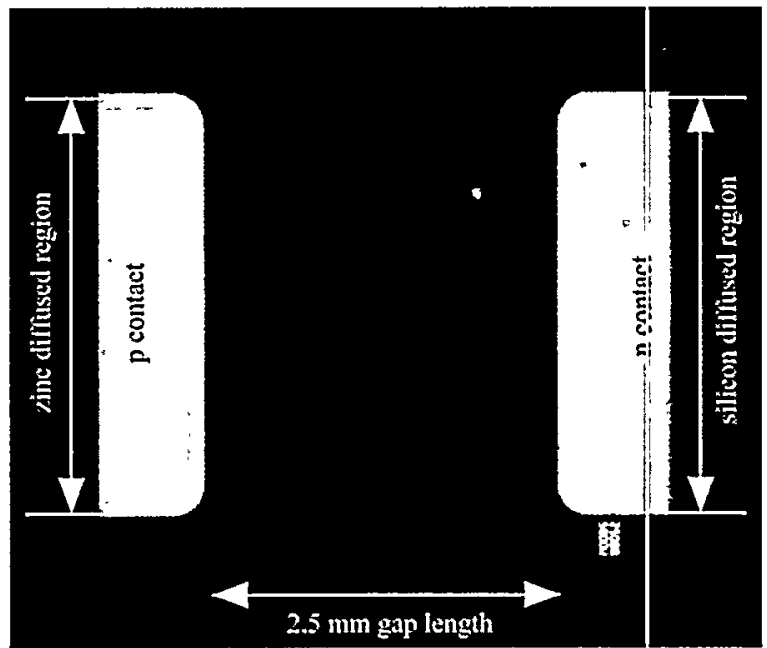

Figure 3. Microphotograph of completed PCSS with diffusion-doped contacts.

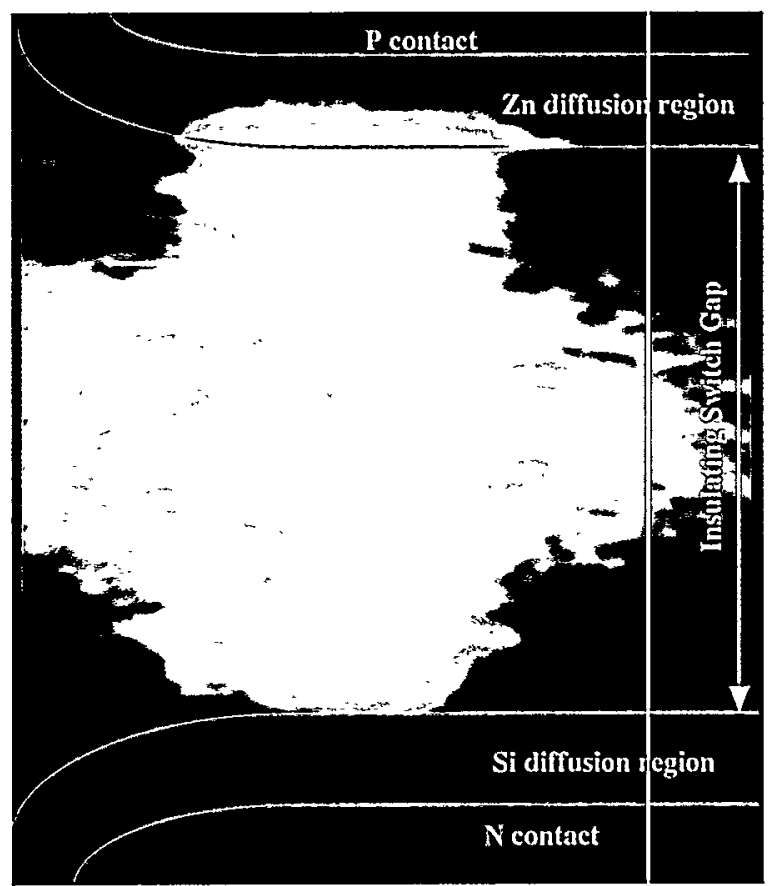

Figure 4. Filament suppression in the doped contact regions of a PCSS.

constant even if the metal contact is damaged, until the point where the external connection to the device is destroyed.

The level of current that can be switched without damage is dependent on the thickness of the doped layer beneath the contact. This was determined by preparing samples over a range of p-type anode diffusion depths (from $\sim 1 \mu \mathrm{m}$ to $\sim 4 \mu \mathrm{m}$ ). The maximum current for operation with no detectable damage was then determined 


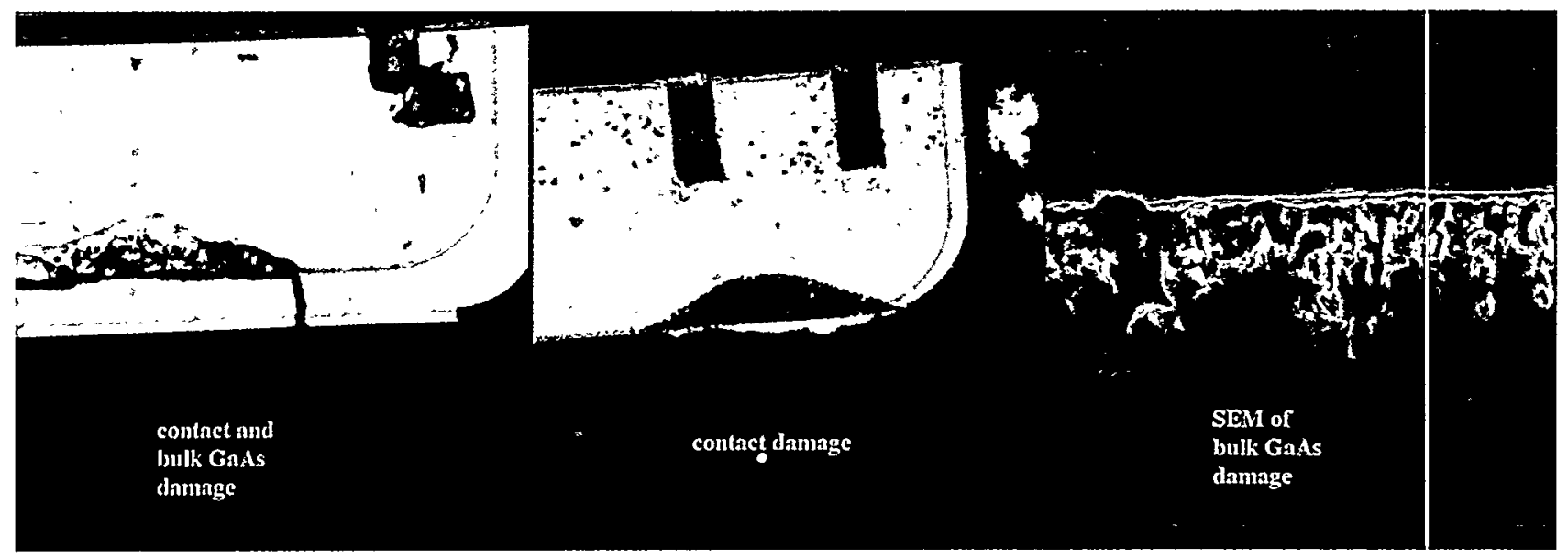

Figure 5. Different types of damage to PCSS contacts.

for the different diffusion depths. This was done accounting for two types of damage: damage confined to the contact metal (no switching current degradation), and damage to both the contact metal and bulk GaAs (switching current drops as damage accumulates). This characteristic is plotted in Figure 6 . It is clearly seen that a thicker doped layer aids in suppressing damage to the bulk semiconductor. However, the improvement for damage to the contact metal tends to a limit of approximately $40 \mathrm{~A}$, with no further improvements with increasing diffusion depth. This implies a different mechanism for damage to the metal that is not adequately addressed by the current spreading in the doped contact layer. This indicates the requirement for a separately engineered solution for the fabrication of the contact metals, together with the doping in the semiconductor

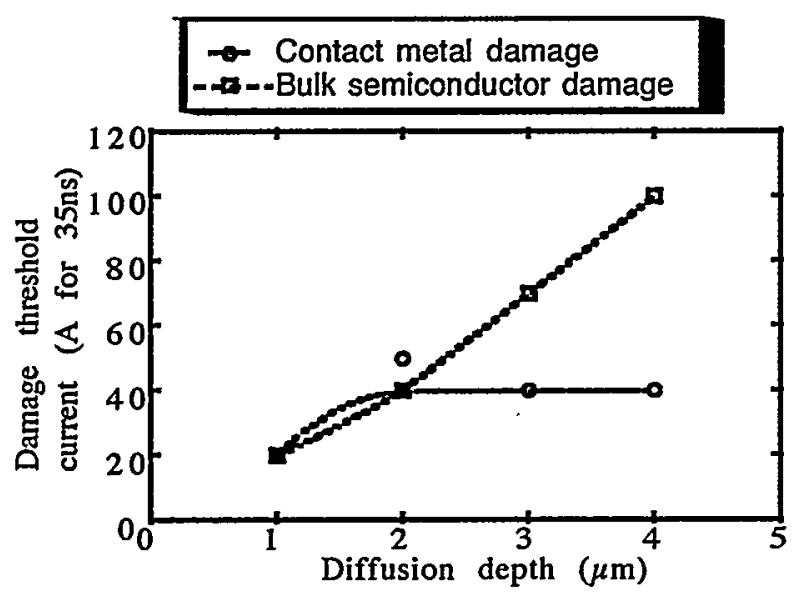

Figure 6. Effect of p-type dopant diffusion depth on maximum damage-free operating current. material, or the implementation of field shaping to enhance the lateral spreading of current at the contact. Beyond such an improvement, the data indicate the need for yet deeper diffusion depths to increase the inherent current capacity of the switch.

\section{LATERALLY-TRIGGERED VERTICAL SWITCH WITH DOPED CONTACTS}

Non-coplanar switch geometries may offer sorne benefit for PCSS longevity. We are currently developing a vertical switch that also incorporates doped contact layers on both sides of the substrate. This eliminates the need for growth patterning or etching. A disadvantage of this approach has been the difficulty in injecting the trigger light into the middle of the switch gap. I3y using a cleaved surface for triggering, we are able to trigger this device the same manner as the co-planar switch, as shown in Figure 7. This allows us to retain the advantage of controlling the filament formation across the entire gap with the trigger light. Varying the size of the switch gap may be accomplished through the use of different wafer thicknesses or by stacking. This geometry combines the advantages of lateral switches with doped contacts of infinite effective depth orthogonal to the filament. The initial testing of this switch shows damage-free operation at currents of up to at least 50A.

\section{APPLICATION TO FIRING SETS}

For firing set applications we are interested in currents that range from $80 \mathrm{~A}$ to $3 \mathrm{kA}$ and pulse durations that are as high as a few $100 \mathrm{~ns}$ with a requirement for lifetime of less than a few hundred shots. At the high current: levels, 


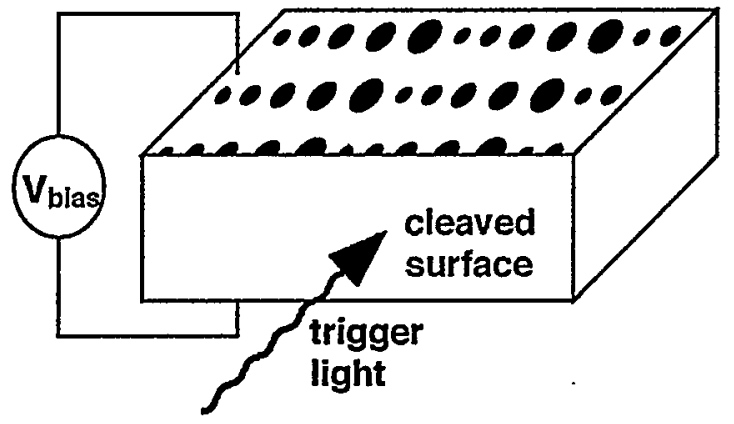

P- contact epitaxy: $2 \mu \mathrm{m}$ thick $\mathrm{p}^{+}$GaAs $\mathrm{N}$ - contact epitaxy: $2 \mu \mathrm{m}$ thick $n^{+}$GaAs

Figure 7. Laterally triggered vertical switch with top and bottom doped contacts.

the doped contact structure does not adequately suppress filament formation to prevent contact damage when a single spot trigger is used. One way to achieve increased longevity is to utilize multi-line triggering to spread the current into multiple filaments such that the current carried by a single filament is sufficiently low for the filament suppression in the doped material.

Fireset switches are generally neutron bombarded to enhance radiation hardness and high voltage holdoff. Another longevity issue for these switches is the longterm stability of the electrical properties of irradiated GaAs due to "self-annealing" of the neutron-induced damage. Figure 8 shows the time dependence of the DC voltage required to induce $50 \mu \mathrm{A}$ leakage current in an irradiated PCSS. Although relatively preliminary, the data indicate that the enhanced DC holdoff, will likely remain adequate for decades.

\section{SUMMARY AND FUTURE DIRECTIONS}

The incorporation of a doped layer beneath PCSS contacts is shown to be very effective in the suppression of filament formation and alleviating current crowding to improve the longevity of PCSS. Damage-free operation is now possible at much higher current levels than before. We have now obtained over 100 million shots from a PCSS at 23A. At 80A, 2 million shots have been obtained with no detectable damage. The inherent current carrying capacity for no damage in the bulk GaAs depends on the thickness of the doped layers and is at least $100 \mathrm{~A}$ for a dopant diffusion depth of $4 \mu \mathrm{m}$. Further independent improvement of the contact metal itself

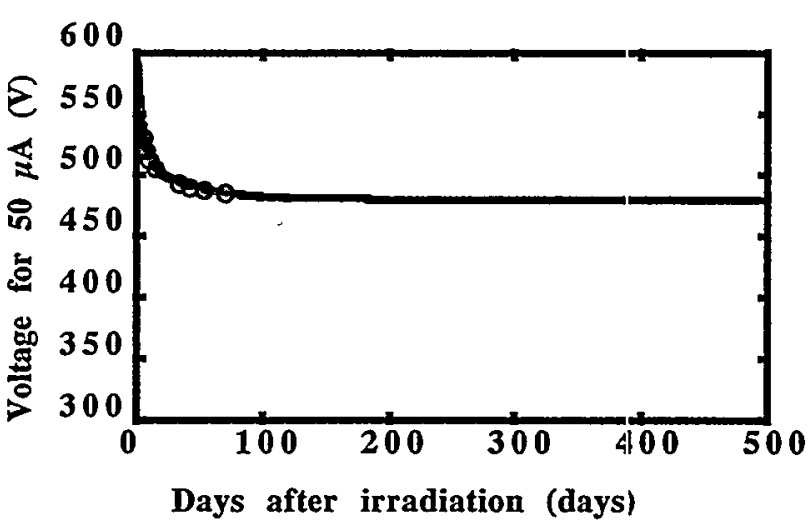

Figure 8. Projected time dependence of $D C$ leakage current in a neutron-bombarded fireset PCSS.

and/or multiple filament triggering is necessary to obtain further overall improvements in longevity. Howrever, in a doped contact switch, the switching performance is not degraded when contact metal erosion occurs.

Sandia is a multiprogram laboratory operated by Sandia Corporation, a Lockheed Martin Company, for the United States Department of Energy under Contract DEAC04-94AL85000.

\section{REFERENCES}

[1] G. M. Loubriel, M. W. O'Malley, and F. J. Zutavern, "Toward pulsed power uses for photoconductive semiconductor switches: closing switches," Proc. 6th IEEE Pulsed Power Conf., P. J. Turchi and B. H. Bernstein, eds., (IEEE, NY, 1987), Arlington, VA, June 29-July 1, 1987, pp. 145-148.

[2] F. J. Zutavern, G. M. Loubriel, M. W. O'Nlalley, W. D. Helgeson, and D. L. McLaughlin, "High Gain PCSS," Proc. 8th IEEE Pulsed Power Conference, R. White and K. R. Prestwich, eds., San Diego, CA, 1991, p. 2:.

[3] G. M. Loubriel M. Loubriel, F. J. Zutaverr, A. Mar, M. W. O'Malley, W. D. Helgeson, D. J. Brown, H. P. Hjalmarson, and A. G. Baca, "Longevity Of Optically Activated, High Gain GaAs Photoconductive Semiconductor Switches," Proc. 10th IEEE Pulsed Power Conf., (IEEE, NY, 1997).

[4] J. W. Scott, R. S. Geels, S. W. Corzine, and L. A. Coldren, "Modeling Temperature Effects and Spatial Hole-Burning to Optimize Vertical-Cavity SurfaceEmitting Laser Performance," IEEE J. of Quantum Elec., Vol. 29 (\#5), pp. 1295-1308, 1993.

[5] R.L. Thornton, R.D. Burnham, T. L. Paoli, N. Holonyak Jr., and D. G. Deppe, "Optoelectronic device structures fabricated by impurity-induced disorclering", $J$. Crystal Growth 77, pp. 621-628, 1986. 